\title{
Activity of Intracellular Metabolism at Neutrophils in Patients with Metabolic Syndrome
}

\author{
Andrey Eu. Kratnov \\ Head of the Department of Therapy of the Paediatric Faculty of Yaroslavl State Medical Academy, \\ Yaroslavl, Russia \\ kratnov@mail.ru
}

Received date: 23 October 2013, Accepted date: 29 January 2014, Published date: 14 February 2014

Academic Editor: Carla Roberta Oliveira Carvalho

Copyright (C) 2014. Andrey Eu. Kratnov. Distributed under Creative Commons CC-BY 3.0

\begin{abstract}
There have been studied factors of metabolic syndrome, risk of development of type 2 diabetes on test-questionnaire "FINDRISK", and parameters of intracellular metabolism of neutrophils in 94 men (mean age $47.7 \pm 7.4$ years) without ischemic heart disease. Increased risk of developing type 2 diabetes in patients with metabolic syndrome was accompanied by growth activity of myeloperoxidase and contents of hydrogen peroxide in neutrophils, and also increased body mass index. In patients with expressed obesity III, the growth level of oxygen-dependent metabolism of neutrophils was observed on the background of decreasing antioxidant defence in phagocytes that can promote the development of oxidative stress.
\end{abstract}

Keywords: metabolic syndrome, neutrophils, oxidative stress.

\section{Introduction}

In recent years the spread of the metabolic syndrome, the presence of which is accompanied by increased risk of developing type 2 diabetes mellitus (DM) and cardiovascular disease has taken epidemic proportions [4]. There is evidence that metabolic syndrome is accompanied by inflammation of adipose tissue, which manifests its cellular infiltration and secretion of adipokines change. It is believed that one of the adipokines - leptin activates neutrophils and macrophages, stimulating in them cytokine secretion, enhances the expression of adhesion molecules and contributes to the development of oxidative stress [13].

The aim of this study was to investigate the intracellular metabolism of neutrophils in patients with metabolic syndrome without coronary heart disease (CHD), depending on the risk of developing diabetes.

\section{Methodology}

94 men aged 29 to 64 years (mean age 47.7 \pm 7.4 years) have been examined. In order 
to exclude CHD in all patients electrocardiography (ECG) was performed, along with bicycle ergometry, Holter ECG monitoring, and echocardiography. The diagnosis of metabolic syndrome criteria were used by experts from the International Diabetes Federation with the participation of the U.S. National Institute of Heart, Lung, and Blood (2009) [1]. Patients with diabetes were excluded. Metabolic syndrome was diagnosed in 78 (82.9\%) patients studied. In individuals with metabolic syndrome 10-year risk of DM was assessed by test-questionnaire "FINDRISK" expert endocrinologists Society of Finland. High 10-year risk of developing diabetes (total score $\geq 15$ ) was observed in 32 (41\%) patients with metabolic syndrome.

For the diagnosis of obesity, body mass index (BMI) was calculated. Obesity was encountered in $74(78.7 \%)$ evaluable patients, of whom $46(62.2 \%)$ revealed I degree of obesity (BMI $30-34.9 \mathrm{~kg} / \mathrm{m}^{2}$ ), 21 (28.4\%) - II degree of obesity (BMI 35-39.9 $\left.\mathrm{kg} / \mathrm{m}^{2}\right)$, and 7 (9.4\%) patients - III degree of obesity (BMI $\geq 40 \mathrm{~kg} / \mathrm{m}^{2}$ ).

The material for this study was peripheral blood analysis which was performed at admission of patients to the hospital with the consent of the Ethical Committee. Neutrophils were isolated from heparinised blood in Ficoll-Verographin double density gradient 1.077 and 1.092 $\mathrm{g} / \mathrm{cm}^{3}$. The second interface cells contained $95 \%$ of neutrophils. The number of neutrophils in the cell suspension was counted in a cell with Goryaev vivo staining with methylene blue in $3 \%$ acetic acid (Turk dye) to determine viable cells. Viability of phagocytes, estimated by trypan test was more than $90 \%$. In order to achieve a concentration of $5 \times 10^{6}$ neutrophils in $1 \mathrm{ml}$ of cell suspension was diluted with medium 199.

The test of spontaneous and stimulated nitro blue tetrasolium reduction was performed by means of quantitative spectrophotometric method using $0.2 \%$ nitro blue tetrazolium in phosphate buffer, fixing neutrophils with methanol and dissolving in mixture of reduced diformazan potassium hydroxide and dimethylsulfoxide 3:5 volume mixture [3].

Myeloperoxidase activity in neutrophils was studied using a $0.04 \%$ solution ortofenilendiamin in phosphate buffer $\mathrm{pH}$ 5.0 , with the addition of $0.33 \%$ hydrogen peroxide solution at a ratio of $20: 1$ by volume. The reaction was stopped after 10 minutes of $10 \%$ sulphuric acid solution. Photometry was carried out at $\lambda=492 \mathrm{nM}$ [12].

The determination of hydrogen peroxide in neutrophils was carried out by a quantitative spectrophotometric method using $0.2 \%$ solution of phenol red. The method is based on the ability of hydrogen peroxide to form an unidentified product during the interaction with the phenol red. After incubation for $60 \mathrm{~min}$ at $37^{\circ} \mathrm{C}$, the reaction was stopped by adding $1 \mathrm{M}$ solution of $\mathrm{NaOH}$. The color intensity was measured at $\lambda=600 \mathrm{nM}$ against a control sample in which the reaction was stopped immediately after addition of the dye [8].

The determination of catalase activity in neutrophils was based on the ability of hydrogen peroxide to form salts with molybdenum stable colored complex. The reaction was run by adding $0.03 \%$ hydrogen peroxide solution and was stopped after 10 minutes, adding a 4\% solution of ammonium molybdate. In control test instead of neutrophils distilled water was brought. The color intensity was measured at $\lambda=410 \mathrm{nM}$ against a control sample of distilled water [7].

Statistical data processing was performed using parametric and nonparametric methods, using the package Statistica 8.0 (StatSoft, Inc., USA). These studies are presented in the form of their averages and standard deviations $(M \pm S D)$. Differences between groups were considered statistically significant at $p<0.05$.

\section{Results}

Patients with high 10-year risk of developing diabetes, compared with other persons with the metabolic syndrome were significantly older $(50.2 \pm 7.6>45.2 \pm 6.3$ 
years, $p=0.002$ ) and had higher levels of glucose capillary blood $(5.5 \pm 0.6>4.7 \pm$ $0.3 \mathrm{mmol} / 1, p=0.00001)$. In the study of intracellular metabolism of neutrophils was identified (Figure 1) that these patients had higher myeloperoxidase activity $(13.1 \pm 15.4>6.8 \pm 6.4$ Sigma units, $p=0.01$ ), and doubtfully the content of hydrogen peroxide $(52.6 \pm 25.8>45.1 \pm$ 19.8 units of optical density, $p=0.09$ ) in phagocytes. In patients with high 10-year risk of developing diabetes (Figure 2) body mass index $(34.8 \pm 5.1>32.9 \pm 4.2$ $\mathrm{kg} / \mathrm{m}^{2}$ ) was higher.

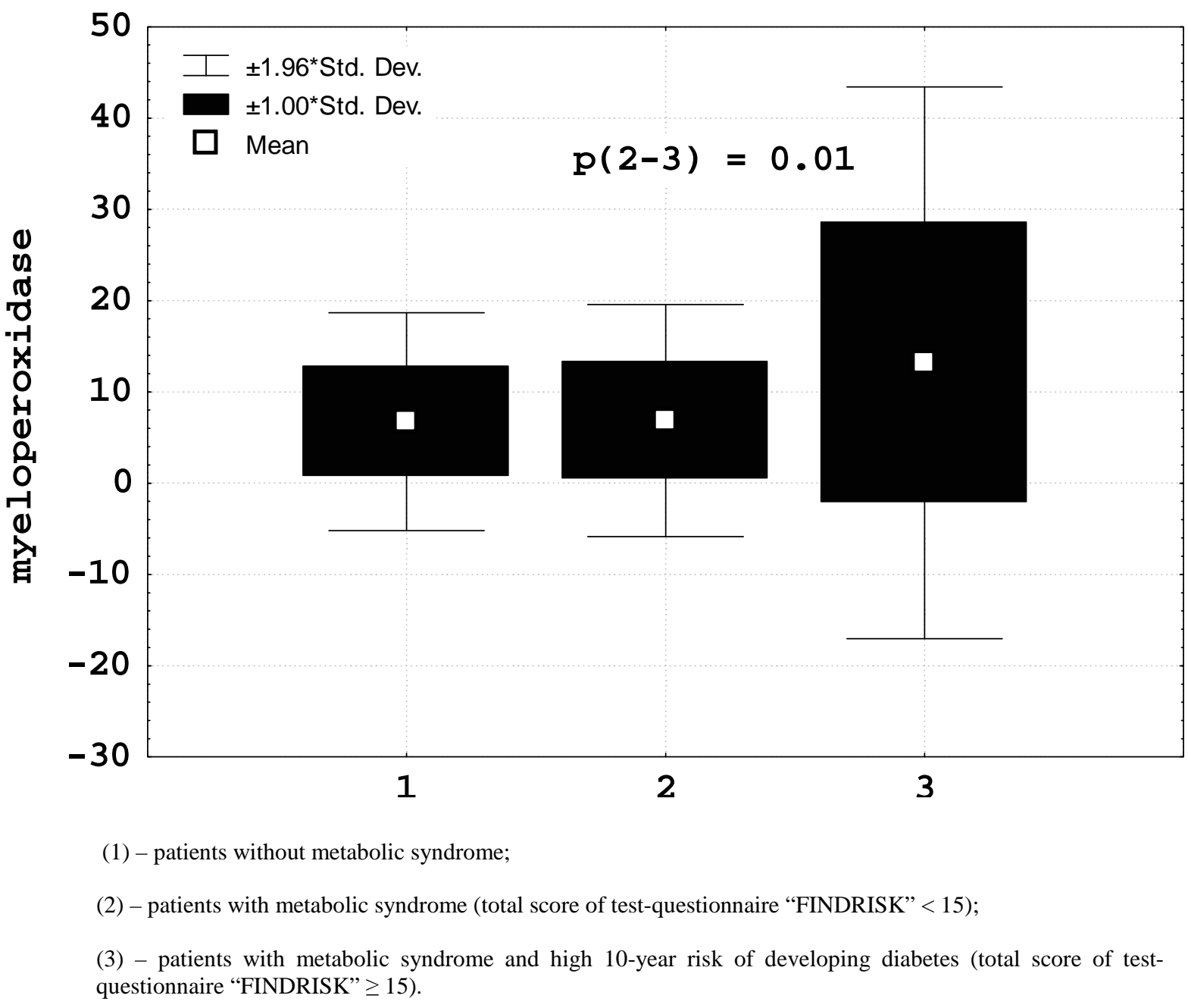

Figure 1. Myeloperoxidase activity at neutrophils in patients with metabolic syndrome depending on risk of developing type 2 diabetes mellitus. 


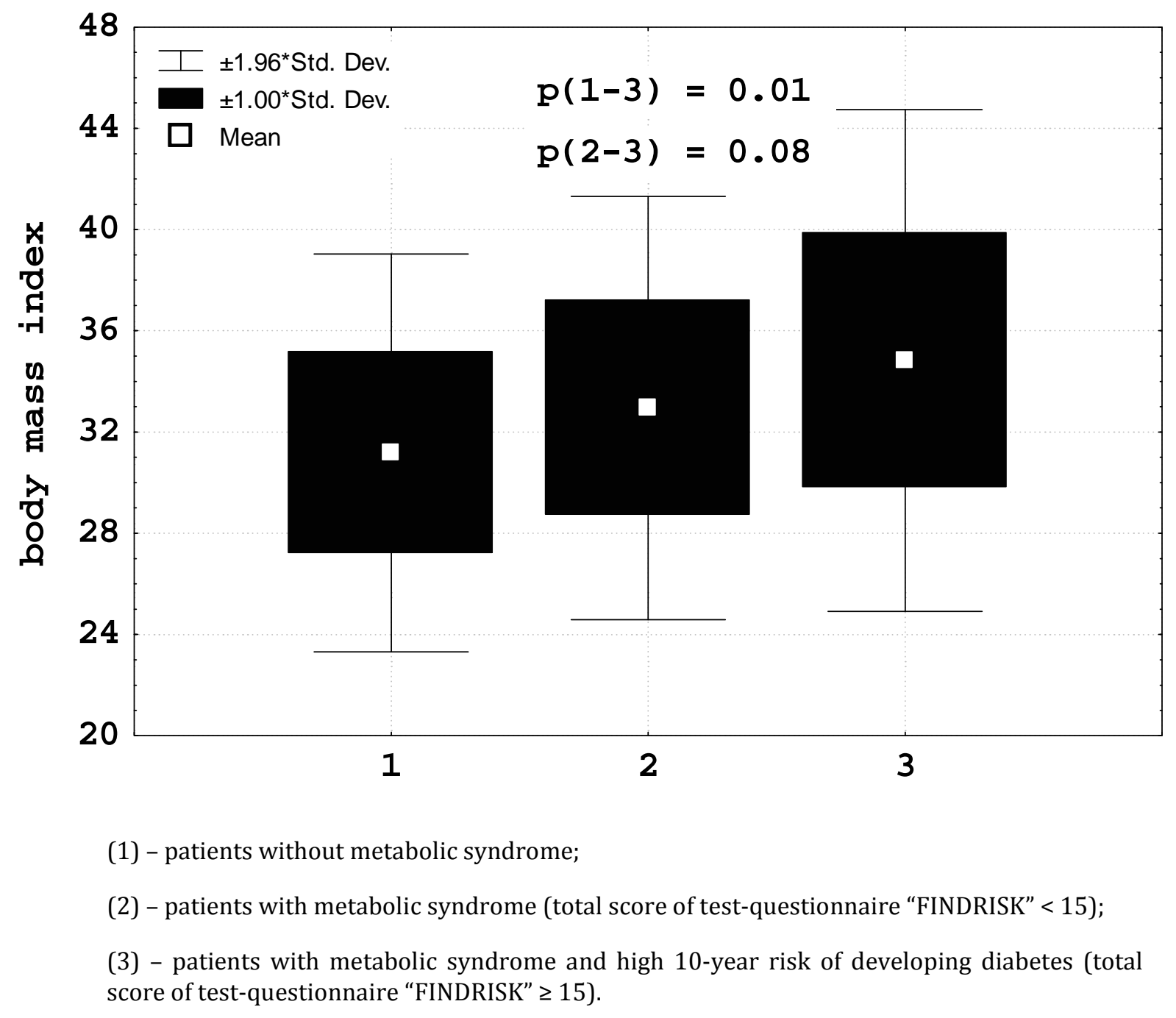

Figure 2. Body mass index in patients with metabolic syndrome depending on risk of developing type 2 diabetes mellitus.

The study of intracellular metabolism of neutrophils in the metabolic syndrome has revealed that its changes are due to the patients of general obesity. In patients with obesity III level, compared with other persons, revealed an increase in myeloperoxidase activity $(12.5 \pm 10.1>8.7$ \pm 10.1 Sigma units) in neutrophils. The growth of oxygen-dependent metabolism of neutrophils in patients with severe obesity (Figure 3) was accompanied by a significant decrease in the intracellular activity of catalase $(190.8 \pm 167.5<315 \pm$ $115.9 \mathrm{mCat} / \mathrm{l}, p=0.01)$. In patients with high risk of developing diabetes, significant negative correlation ( $\mathrm{r}=-0.51, \mathrm{p}<0.05)$ between body mass index and catalase activity in neutrophils was observed (Figure 4). 


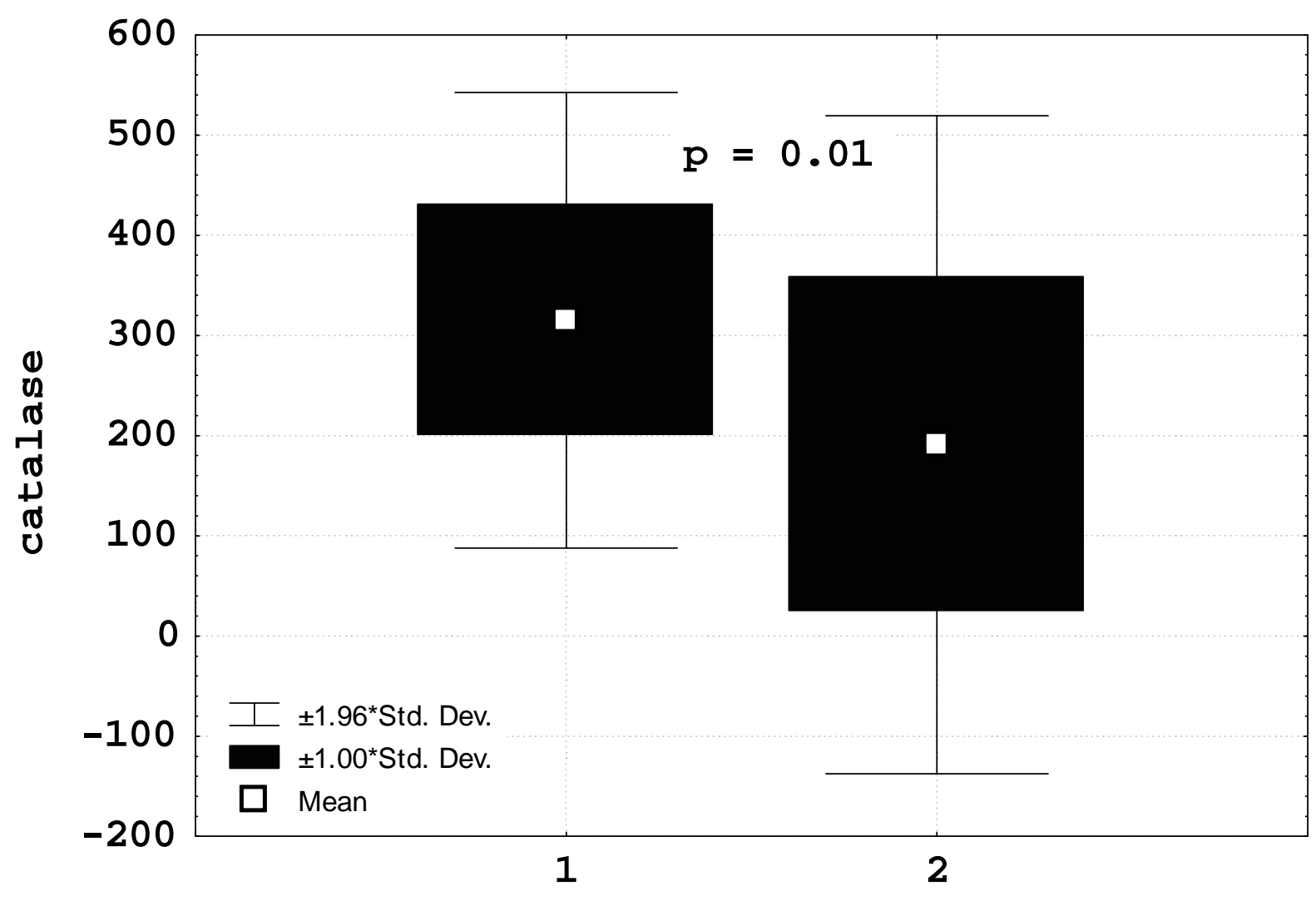

(1) - patients with body mass index $<40 \mathrm{~kg} / \mathrm{m}^{2}$;

(2) - patients with body mass index $\geq 40 \mathrm{~kg} / \mathrm{m}^{2}$.

Figure 3: Catalase activity at neutrophils depending on body mass index. 


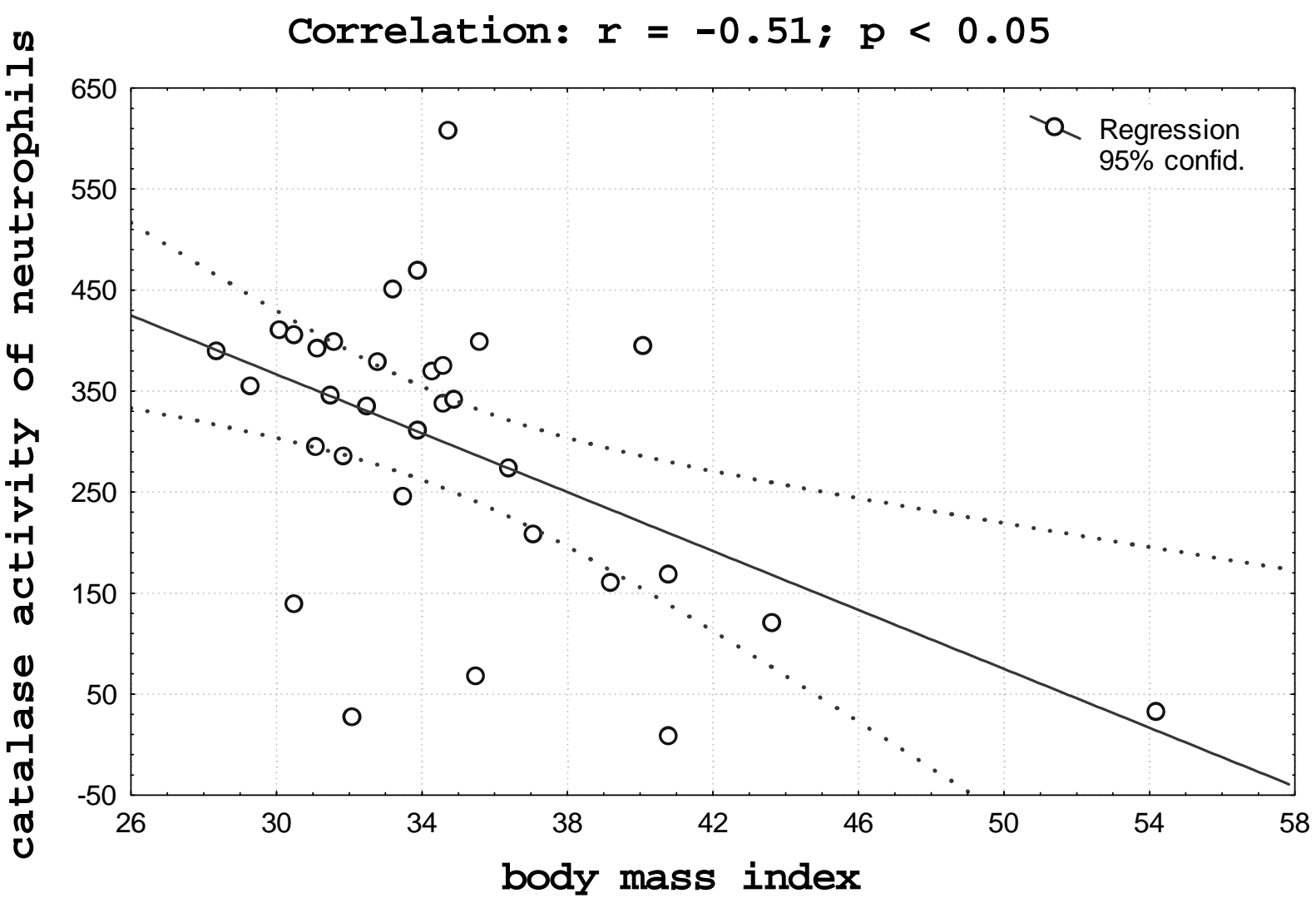

Figure 4. Correlation between body mass index and catalase activity at neutrophils in patients with high risk of developing type 2 diabetes mellitus.

\section{Discussion}

The main argument of increased interest in the metabolic syndrome is its association with atherosclerosis and the risk of cardiovascular disease. Revealed obesity and hyperglycemia correlated with the presence of atherosclerotic plaques in coronary arteries and abdominal aorta in young people aged 15-34 years, were found during post-mortem autopsy [14]. It is shown that the presence of the metabolic syndrome is accompanied by increased blood levels of proinflammatory cytokines, resulting probably in adipose tissue, and stimulating the formation of C-reactive protein. It is known that $\mathrm{C}$-reactive protein enhances the mobility of leukocytes, initiates phagocytosis and binding of complement, and stimulates expression of adhesion molecules on the surface of the endothelium $[9,10]$. In recent years, much importance is given to the presence of reactive oxygen species in the development of complications, and to the occurrence of cardiovascular events in diabetic patients [15]. The activation of oxygen-dependent metabolism of neutrophils to the development of an adverse outcome in patients with CHD was previously established [5].

The present study revealed that the increased risk of diabetes in patients with metabolic syndrome is accompanied by increased oxygen-dependent metabolism neutrophils, activation of which is also associated with obesity. It was found those patients with high 10-year risk of diabetes increases myeloperoxidase activity and the content of hydrogen peroxide in neutrophils, which are capable of forming enzyme-substrate complex. The most toxic products of myeloperoxidase are 
hypochlorous acid and hypochlorite anion, which provide the main belongings to the antimicrobial function of neutrophils. Hydrogen peroxide and hypochlorous acid can decompose to form reactive hydroxyl radical, which is the major damaging agent in biological systems [2].

It was revealed that the increased remote cardiovascular risk as the risk of death from all causes, has been observed mainly in cases of severe obesity [11]. In the present study, in patients with obesity III degree increased content of hydrogen peroxide in neutrophils was observed against the background of a significant reduction in activity of intracellular antioxidant protection. It was previously shown that a decrease of antioxidant defence in neutrophils is accompanied by an increased risk of death in CHD [6]. It can be assumed that the proinflammatory state observed in the metabolic syndrome has the ability to contribute to the instability of the atherosclerotic plaque and thus predispose to the development of cardiovascular events.

\section{Conclusion}

Increased risk of diabetes in patients with metabolic syndrome is accompanied by increased oxygen-dependent metabolism of neutrophils; activation accompanied by decreasing antioxidant defence in phagocytes is associated with obesity.

\section{References}

1.Alberti, K. G., Eckel, R., Grundy, S. M., Zimmet, P. Z., Cleeman, J. I., Donato, K. A., Fruchart, J. C., James, W. P., Loria, C. M. and Smith, S. C. (2009) "Harmonizing the metabolic syndrome: a joint interim statement of the International Diabetes Federation Task Force on Epidemiology and Prevention; National Heart, Lung, and Blood Institute; American Heart Association; World Heart Federation; International Atherosclerosis Society; and International Association for the Study of Obesity," Circulation, 120 (16) 1640-1645.
2.Babior, B. M. (1984) "Oxidants from phagocytes: agents of defence and destruction," Blood, (64) 959-967.

3.Gentle, T.A. and Thompson, R. A. (1990) Neutrophil function tests in clinical immunology. In: Gooi HG, Chapel H. Clinical immunology. A practical approach (2nd ed.), Oxford University Press, New York.

4.Isomaa, B., Almgren, P., Tuomi, T. and Forsen B. (2001) "Cardiovascular morbidity and mortality associated with the metabolic syndrome," Diabetes Care, 24 (4) 683-689.

5.Kratnov, A. E. (2002) “Oxygen-dependent phagocyte metabolism and blood plasma antioxidant defence in acute coronary syndromes depending on the outcome during hospitalization," Klin. Lab. Diagn., (6) 6-13.

6.Kratnov, A. E. and Khabarova, I. V. (2009) "Neutrophilic intracellular metabolic changes and fatal outcome in coronary heart disease," Klin. Lab. Diagn., (1) 20-22.

7.Mamontova, N. S, Beloborodova, E. I. and Tucalova, L. I. (1994) "Activity of catalase at chronic dipsomania," Klin. Lab. Diagn., (1) 27-28.

8.Pick, A. and Keisari, Y. (1981) "Superoxide anion and hydrogen peroxide production by chemically elicited peritoneal macrophages," Cellular Immunol., 59 (2) 301-318.

9.Ridker, P. M., Buring, J. E., Cook, N. R. and Rifai, N. (2003) "C-reactive protein, the metabolic syndrome, and risk of incident cardiovascular events: an 8-year follow-up of 14719 initially healthy American women," Circulation, 107 (3) 391-397.

10.Ridker, P. M., Wilson, P. W. and Grundy, S. M. (2004) "Should C-reactive protein be added to metabolic syndrome and to assessment of global cardiovascular risk?" Circulation, (109) 2818-2825.

11.Romero-Corral, A., Montori, V., Somers, V. K., Korinek, J., Thomas, R.J., Allison, T.G., Mookadam, F. and Lopez-Jimenez F. (2006) "Association of bodyweight with total 
mortality and with cardiovascular events in coronary artery disease: a systematic review of cohort studies," Lancet, 368(9536) 666-678.

12.Saidov, M. Z. and Pinegin, B. V. (1991) "Spectrophotometric analysis of myeloperoxidase activity in phagocytes," Laboratornoe delo, (3) 56-59.

Schwarz, V. (2009) "Inflammation of adipose tissue and atherosclerosis," Kardiologiia, 49 (12) 80-86.

13.Strong, J. P., Malcom, G. T., McMahan, C. A., Tracy, R. E., Newman, W. P. III,
Herderick, E. E. and Cornhill, J. F. (1999) "Prevalence and extent of atherosclerosis in adolescents and young adults: implications for prevention from the Pathobiological Determinants of Atherosclerosis in Youth Study," JAMA, 281 (8) 727-735.

14.Ziegler, D., Sohr, C. G. H. and NouroozZadeh, J. (2004) "Oxidative stress and antioxidant defence in relation to the severity of diabetic polyneuropathy and autonomic neuropathy," Diabetes Care, (27) 2178-2183. 